\title{
Methods to Combine Multiple Images to Improve Quality
}

\author{
Anders. P. Kaestner \\ ${ }^{1}$ Laboratory for Neutron Scattering and Imaging, Paul Scherrer Institut, CH5232 Villigen-PSI, \\ Switzerland \\ anders.kaestner@psi.ch
}

Keywords: Outliers, Image Combination, Neutron Imaging

\begin{abstract}
Noise and artifacts have a negative impact on the image quality and the resulting image analysis. The signal to noise ratio (SNR) caused by the neutron flux and the light conversion efficiency is one component in this. Here, we are more concerned about the effect of outliers, which frequently appear in neutron images. There are two approaches to reduce the impact of outliers (1) by applying spatial outlier rejection filters on each image and (2) by acquiring multiple images which are combined into a single image with a total neutron dose similar to the dose of the image in option (1). Here, we focus on the second option where we will show the importance of the choice of combination method. The impact is demonstrated to show the ability to reject outliers but also that the SNR can be improved. The image combination approach has the advantage that it does not affect neighbor pixels or larger regions. The tested methods are the arithmetic average, median, and a new weighted average. The weighted average shows promising results compared to the other two alternatives both regarding improving SNR and its outlier rejection ability.
\end{abstract}

\section{Introduction}

Neutron imaging is often performed at relatively low neutron counts. The origin of this is that most neutron sources provide a relatively low flux compared to for example X-ray sources. The provided neutrons are used in imaging experiments that require high spatial, or temporal resolution, or even both at the same time. The experiment requirements sometimes result in very low neutron counts per pixel in the acquired images. In addition to the Poisson distributed counting noise from neutrons and photons and binominally distributed noise from the acquisition system], it also very common to observe bright outliers in neutron images. The combination of noise and outliers sometimes degrade the images to the degree that makes any reliable analysis impossible. One solution to mitigate this problem is to increase the signal to noise ratio by increasing the exposure times, but this is mostly not realistic due to limited beamtime or limitations that are given by the observed sample. Therefore, numerical methods and alternative acquisition schemes are needed. The outliers are often suppressed using combinations of median filters and outlier detection [1,2]. The counting noise can be reduced by applying different noise reduction filters. In this paper, a different approach is pursued where multiple images are acquired from the same view of the sample. These images are then to be combined into a single image (figure 1). The total exposure time of the combined image shall be equal to an image in the single shot approach. The advantage of this approach is that it introduces an opportunity to reduce noise and outliers without the need to involve pixel neighbors as would be needed when spatial filters are applied. The focus in this paper is, in particular, on different methods to pixelwise combine multiple images into a single image. Three different combination approaches (average, median, and weighted average) will be evaluated using phantom images with different levels of Poisson noise and outlier frequency and strength. Finally, the methods are also demonstrated using real neutron images. 


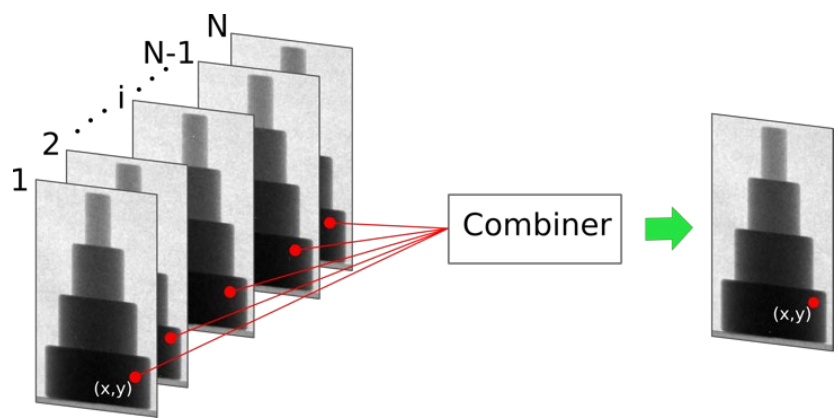

Figure 1: Multiple images are combined pixel-wise into a single image with improved SNR and ideally fewer outliers.

\section{Methods}

The noise and outlier reduction concept investigated in this paper is based on having multiple observations (images) of the same view. These images are to be combined into a single image which ideally shall have higher SNR and fewer outliers than a single image acquired with the equivalent dose as the collection together. Three different combination operations will be evaluated. These are the arithmetic mean (average), median, and weighted average. These operations are to be applied on the image values at the same pixel position in all images in the collection and will provide a new value that is used in the combined image. The operators are defined as follows. With a collection of $N$ 2D images $f_{1} \ldots f_{N}$ the average operator is described as

$$
f_{\text {avg }}(x, y)=\frac{1}{N} \sum_{1}^{N} f_{i}(x, y)
$$

This operator is efficient to improve the SNR for data without outliers; this operator produces a result that corresponds to acquiring a single image with $N$ times longer exposure time. In cases when outliers appear in the data, this operator fails to compute a value corresponding to the expected value. For this reason, the outlier rejecting behavior of the median operator is attractive. This operator is described with

$$
f_{\text {med }}(x, y)=\operatorname{median}\left(\left\{f_{i}(x, y) \mid i \in[1, N], i \in \mathrm{Z}^{+}\right\}\right)
$$

The median is a selection operator that never will be able to obtain an averaging effect. Therefore, the theoretical SNR value is always less than the SNR of the average with outlier free data. A method to combine the advantages of the average (improved SNR) and the median (outlier rejection) and a new method using a weighted average operator is proposed to combine the images

$$
f_{w}(x, y)=\sum_{i=1}^{N} w_{i}(x, y) \cdot f_{i}(x, y)
$$

This averaging is a generalization of (1) which would have $w=1 / N$. The weights based on estimates of the local standard deviation $\hat{\sigma}_{i}$ as

$$
w_{i}(x, y)=\frac{\frac{1}{\hat{\sigma}_{i}(x, y)}}{\sum_{i=1}^{N} \frac{1}{\hat{\sigma}_{i}(x, y)}}
$$


This type of weight penalizes pixels with high values of $\hat{\sigma}_{i}$. High values are typical for pixels with outliers in their neighborhood. If all images at a position have approximately the same value of $\hat{\sigma}_{i}$, they produce weights approaching $w=1 / N$, i.e., the average operation in equation (1). The most efficient way to compute $\hat{\sigma}_{i}$ is to use convolution with box filter kernels of some given size, typically $3 \times 3$ or $5 \times 5$ are used. A $5 \times 5$ kernel was used in the evaluation.

\section{Evaluation}

The performance of the three combination methods was evaluated with a simulation using images with Poisson distributed noise to reflect quantum noise of neutrons arriving at a detector and outliers with different size and strength were added to the noise images. Figure 2 shows examples using increasing number of simulated images in the combination. The blobs visible in the average images show the well-known fact that plain arithmetic averaging is not able reject outliers. In the simulation 25 such image set were created for different signal to noise ratio (SNR) in the different images to study the variation. Two cases were studied: (1) increasing the number of images with constant dose and (2) maintaining the total dose but increasing the number of images in the combination.
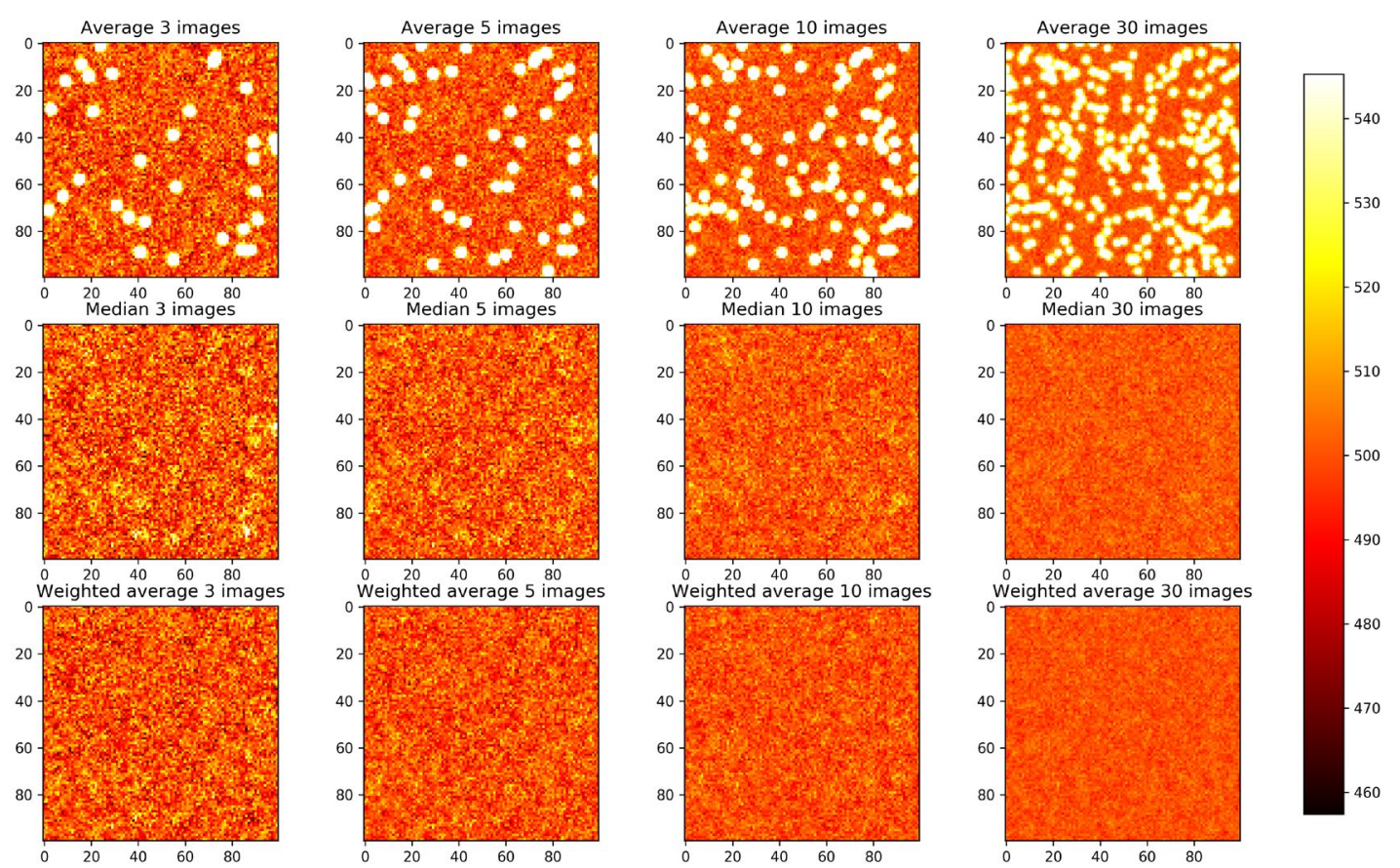

Figure 2: Examples of combination performance using the same simulated test images with constant average gray level with added spots. All images are shown with the same gray level interval. 
In both cases, the simulation shows that the weighted average performs best. The SNR of the processed images was computed as $\mathrm{SNR}=\sigma_{\text {image }} / \mu_{\text {image }}$.
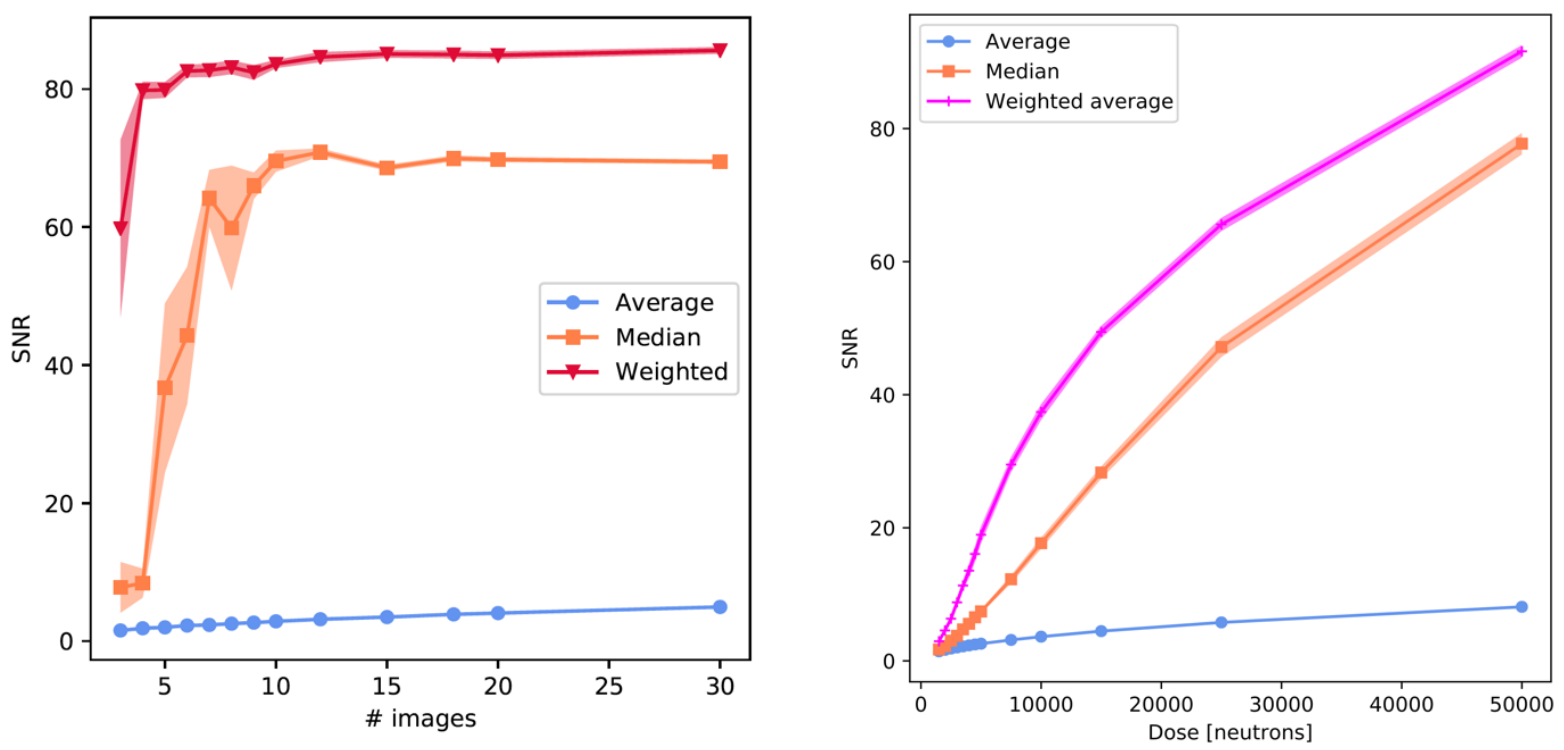

Figure 3: Results from simulations using images with Poisson distributed noise and outliers added. The curves to the left show the performance using a constant total dose split up on multiple images. The curves to the right show the effect of adding more images to the combination and thus increasing the total dose. The error bands correspond to 95\% confidence intervals.

The results of the numeric experiments are plotted in figure 3. The simulations showed improved SNR and spot rejection ability for median and weighted average compared to the average that cannot reject outliers. The difference between median and weighted average is to be expected as the SNR using median is $\propto \sqrt{2 N / \pi}$ [3] for large $N$ compared to $\propto \sqrt{N}$ for averaging methods.

In the example below, the performance of these two methods is demonstrated on measured neutron radiographs. In this example, 3-30 images were combined using the two methods to provide qualitative comparison how each method performs. Both methods can reduce outliers, but the weighted average performs better than the median. There are still some outliers remaining, even when 30 images are combined. These outliers are most likely stuck pixels on the detector and cannot be removed using this method. 

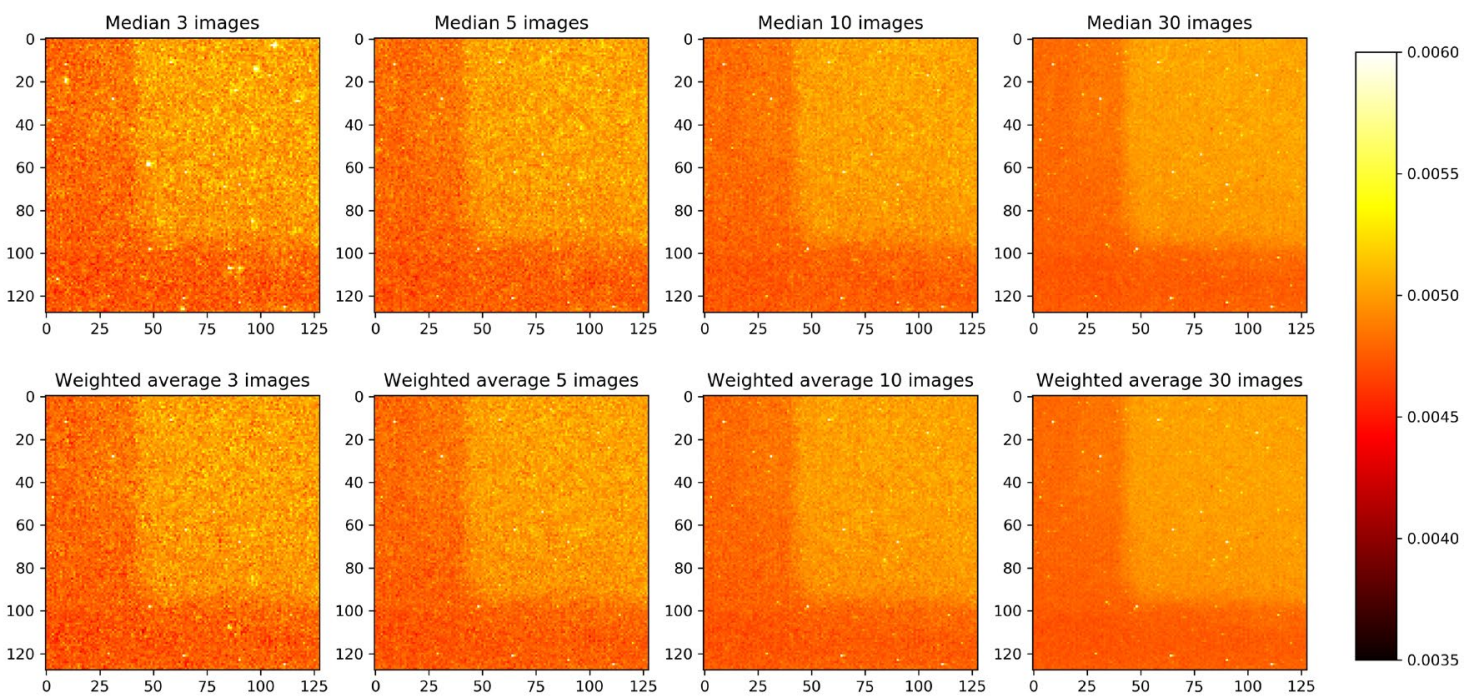

Figure 4: Demonstration of the performance median and weighted average using experimental data.

\section{Summary}

This shows the performance of three different methods to combine images into a single image with the aim to reduce noise and outliers. Simulations indicate that a weighted averaging approach based on local standard deviation outperforms median and arithmetic means as a method to combine several images. The weighted average method has shown promising results both in improving SNR and its ability to reduce the number of outliers. It can also be concluded that image combination as a method to reject gamma spot and to improve the signal to noise ratio. The outlier reducing benefit can already be observed with as few as three images, in particular when the weighted average method is used. In general, both median and weighted average perform best using between five and ten images for the same total dose. A higher number of images will only marginally improve image quality. Therefore, the acquisition overhead and increased storage requirements are not motivated. The presented image combination methods are already implemented in our CT reconstruction tool MuhRec [4].

\section{References}

[1] Hungler, P.; Bennett, L.; Lewis, W.; Bevan, G.; Metzler, J. Comparison of Image Filters for Low Dose Neutron Imaging. Physics Procedia 2013, 43, 169-178.

https://doi.org/10.1016/j.phpro.2013.03.020

[2] Li, H.; Schillinger, B.; Calzada, E.; Yinong, L.; Muehlbauer, M. An adaptive algorithm for gamma spots removal in CCD-based neutron radiography and tomography. Nuclear Instruments \& Methods in Physics Research Section A 2006, 564, 405-413. https://doi.org/10.1016/j.nima.2006.04.063

[3] Weisstein, E.W. CRC Concise Encyclopedia of Mathematics, Second Edition; Chapman and Hall/CRC, 2002. https://doi.org/10.1201/9781420035223

[4] Kaestner, A. MuhRec - a new tomography reconstructor. Nuclear Instruments and Methods A 2011, 212 651, 156-160. https://doi.org/10.1016/j.nima.2011.01.129 